\title{
Determination of Microbiological Salivary Status in Children with Upper Respiratory Tract Disorders
}

\author{
Valë H Hoxha ${ }^{1}$, Agim Begzati², Vlorë H Cakolli ${ }^{3}$
}

\begin{abstract}
Aim: The purpose of this paper is to investigate the prevalence of dental caries and the types of streptococci mutant and Lactobacillus in the saliva of children with middle ear infection (MEI), enlarged tonsils (ET), and healthy children.

Materials and methods: A sample of 93 subjects ( 57 males and 36 females; mean age: $8.1 \pm 1$ years, aged between 5 years and 15 years old) were assessed: 35 patients, with MEI, and 26 with ET, formed the study group, while 32 healthy subjects formed the control group. The participants were interviewed regarding demographic data, dietary, and oral hygiene habits. Their dental caries and oral hygiene status were clinically determined, assessing diagnostic criteria for decayed, missing, and filled teeth for primary (dmft) and permanent (DMFT) dentition, oral hygiene index according to Greene-Vermillion, and the gingival index (GI) according to Silness-Löe. Stimulated salivary flow rate, and salivary mutans streptococci (MS) and lactobacilli (LB) were also determined.

Results: The groups were similar with respect to gender, using fluoride paste, visits to dentist, family income $(p>0.05)$, whereas there was a difference in age, educational level, method of feeding, use of pacifier, sweet eating habits, toothbrushing, saliva secretion, and rate of oral breathing $(p<0.001)$ between intervention and healthy group. However, higher salivary MS and LB levels were observed in MEI, and ET patients compared to controls $(p<0.05)$.

Conclusion: Our results demonstrated that patients with ET and MEI had more initial caries, gingival inflammation, dental plaque, a lower stimulated salivary secretion rate, and an increase in the level of salivary MS and LB than healthy individuals.

Clinical significance: Results from our study will help not only the pediatric dentistry specialists but also the other medical professionals like pediatricians, ENT specialists, and nurses to increase their awareness regarding the importance of the overall dental health in children with an ear infection or tonsillitis.
\end{abstract}

Keywords: Enlarged tonsils, Lactobacilli, Middle ear infection, Mutans streptococci, Oral health.

World Journal of Dentistry (2019): 10.5005/jp-journals-10015-1664

\section{INTRODUCTION}

The association between poor oral hygiene, periodontal disease, and respiratory disease has been increasingly debated over the past several years. ${ }^{1}$ Although the relationship between oral status and pneumonia has been well studied, the association between microorganisms in the oral cavity and upper respiratory tract infections is less clear. ${ }^{2}$ Salivary enzymes associated with periodontal disease may modify the mucosal surfaces along the respiratory tract, thus facilitating colonization by pathogens.' Furthermore, the oral cavity is a potential reservoir for respiratory pathogens. ${ }^{3}$ Middle ear infections diseases of the ET and dental caries are highly prevalent around the globe and are the most commonly diagnosed childhood illnesses. ${ }^{4,5}$ Dental caries in childhood is still a major public health challenge in most industrialized and developing countries. ${ }^{6}$ Previous studies on dental caries demonstrated the role of salivary MS as a predictor and marker for caries risk. ${ }^{7}$ High level of MS in the saliva tends to contribute early colonization of these organisms in children. Children colonized by MS at an early stage developed more caries than those colonized at a later stage. ${ }^{8}$

The regular intake of fermentable dietary sugars, or impaired saliva flow, produces persistent conditions of low $\mathrm{pH}$ within the biofilm, which selects for MS and LB. ${ }^{9}$ Common microbiota of the oral space, MS and LB are accountable for variety of oral illnesses such as caries and periodontitis. ${ }^{10}$ Xerostomia is a common symptom and side effect of respiratory drug therapies that reduce buffering capacity and remineralization ability, leading to elevated caries risk. ${ }^{11}$ Increased severity of the disease and higher numbers

\begin{abstract}
1,2Department of Pediatric Dentistry, School of Dentistry, University Hasan Prishtina, Prishtina, Republic of Kosovo

${ }^{3}$ Department of Periodontology and Oral Medicine, College of Medical Sciences Rezonanca, Prishtina, Republic of Kosovo

Corresponding Author: Vlorë H Cakolli, Department of Periodontology and Oral Medicine, College of Medical Sciences Rezonanca, Prishtina, Republic of Kosovo, Phone: +383 44157885, e-mail: vlora.cakolli@gmail.com How to cite this article: Hoxha VH, Begzati A, Cakolli VH. Determination of Microbiological Salivary Status in Children with Upper Respiratory Tract Disorders. World J Dent 2019;10(5):327-334.
\end{abstract}

Source of support: Nil

Conflict of interest: None

of therapeutic medications are associated with increased caries prevalence. $^{12}$

The aim of this research was to investigate the oral hygiene habits, gingival condition and dietary habits, salivary levels of MS and LB among MEI, ET, and healthy children.

\section{Materials and Methods Subjects}

The study was carried out at the Clinical Center for Pediatric and Preventive Dentistry of the University Dentistry Clinical Center of Kosovo in Pristina, ENT Clinic and the Department of Microbiology of the National Institute of Public Health of Kosovo in the period 2017/2018. The study model is prospective comparative type. 
In this research a sample of 93 children with ET group $(n=35)$, middle ear infected group $(n=26)$, and a healthy children group $(n=32)$ were involved, ranging from 5 years to 15 years of age from both genders, males and females.

All of them voluntarily participated in this study and filled out the informed consent form signed by parents. The study was approved by the Research Ethics Committee of the University of Prishtina.

\section{Inclusion and Exclusion Criteria}

Children with ET and otitis media were included in the study group, but not with other health problems, chronic or acute diseases present. Children in the control group-healthy subjects were also without chronic or acute illnesses.

The study did not include patients with acute health problems of the cardiovascular system, acute and chronic pulmonary disease, gastrointestinal system disorders, metabolic disease, kidney failure, blood discrepancy, and those who did not sign the patient consent form.

All children who participated in the study were examined with dental instruments for examination using artificial light seated in dental chair.

Before dental treatment, a diagnosis of MEl and ET was based on the physical examination by an otolaryngologist (ENT specialist).

For this paper, a special study questionnaire was created, which contains:

The general (demographic) data of the patient [name and surname, gender, age, child's residence and complete medical history, education, familial economic status, nutritional background (breastfeeding vs dietary milk intake), hygiene habits (tooth brushing practices, usage of pacifier, fluoride therapy, oral breathing, and the extent of the saliva secretion)]; were acquired from their parents using a questionnaire.

Determination of dental status was done utilizing the WHO (World Health Organization) criteria ${ }^{13}$ by means of decay-filled teeth (dft) for primary teeth and the decay-missing-filled teeth (DMFT) index for permanent teeth. Furthermore, the DMFT score for any subject may range from 0 to 32 , followed by the dft score, which may range from 0 to 20 in total numbers. ${ }^{14}$

Gingival index, according to Löe-Silness, ${ }^{15}$ has been used to evaluate the gingival status (changes in color, size, and bleeding). The evaluation is done using the exploratory probe, after drying the dental surfaces, with compressed air. All necessary instruments were sterilized before the examinations. The GI values are marked 0 to 3 where: 0 -healthy sound: the gingiva has a light pink color, with grain structure, the papillae are in the interdental space, and no signs of the protrusion. The shape of the papilla depends on the shape of the tooth; 1 -light inflammation: the gingival lining is slightly reddish, has slight edema, and increased gingival exudate. The gingiva does not bleed in the probe with a dental instrument; 2-average inflammation: the gingiva is reddish, it has expressed edema and increased gingival limbus, and there is bleeding after the probe, and 3-severe inflammation: the gingiva is very reddish and grown.

For the purpose of this study, oral hygiene status was assessed using the Greene and Vermillion Simplified Oral Hygiene Index (OHI-S). ${ }^{16}$ The six surfaces of the four posteriors and two anterior teeth were stained with basic fuchsine and examined later. In the posterior portion of the dentition, the first fully erupted tooth, usually the first molar (No. 16), is examined. The buccal surfaces of the selected upper molar and the lingual surfaces of the selected lower molar were evaluated. In the anterior region of the mouth, the central incisors, specifically the labial surfaces of the upper right incisor, No. 11, and the lower-left incisor, No. 31, were scored. OHI-S values may range from 0 to 3 as described: $\mathrm{OHI}-\mathrm{S} 0=$ no debris or pigment present. $\mathrm{OHI}-\mathrm{S} 1=$ soft residue covering no more than one-third of the tooth surface or the presence of surface stains with no other debris, irrespective of the affected area covered; $\mathrm{OHI}-\mathrm{S} 2$ = soft residue overlapping more than one-third but not more than two-thirds of the exposed tooth surface. OHI-S $3=$ soft residues covering more than two-thirds of the exposed tooth surface. Results of $0-1$ are presented as low, and 2-3 as a high oral hygiene index (OHI-S).

\section{Microbiological Methods for the Identification of Bacterial Species}

After answering the questionnaire, clinical saliva samples were then collected from the children.

The saliva stimulated paraffin tablet was given to children who had to chew for at least 5 minutes and then collected all the saliva created in a scaled glass. After the saliva was collected, the amount of saliva was recorded as the rate of saliva flow per minute ( $\mathrm{mL} /$ minute). Subsequently, a small amount of each saliva sample was used to count bacteria through caries risk test (CRT) (Ivoclar Vivadent Clinical, Schaan, Liechtenstein).

\section{Bacteria Count}

From the scaled glass, filled with saliva from children, a portion of the saliva sample was used to disperse into the culture medium (agar) through pipettes. $\mathrm{A} \mathrm{NaHCO}_{3}$ tablet was placed in the culture agar dish to promote bacterial growth and then stored in an incubator at $35-37^{\circ} \mathrm{C}$ for 48 hours (Fig. 1A). Later, the results obtained were evaluated (Fig. 1B). Streptococci mutant (SM) colonies were presented as small blue groups on blue agar, $<1 \mathrm{~mm}$ in diameter (Fig. 1C), while LB colonies were presented as white colonies on translucent agar (Fig. 1D). The presence of a bacterial count higher than 105 colony forming units (CFU)/mL of saliva specifies an increasing risk of emerging tooth decay. Accordingly, in this research, subjects were separated as SM and LB sums $>$ or $<10^{5} \mathrm{CFU} / \mathrm{mL}^{17}$

We used in total 93 both sided CRT Intro Pack-CRT agar plates. Blue agar surface: determination of MS count in saliva (Fig. 1E), bright agar surface: determination of LB count in saliva (Fig. 1F). In order to carry out this study, for the purpose of screening/ evaluation, four examiners are included.

\section{Statistical Analysis}

Continuous variables were summarized as mean and standard deviation (SD), and the categorical variables were summarized as frequency $(n)$ and percentages (\%). In the statistical analysis, differences between continuous variables were tested with the one-way ANOVA and differences between categorical variables with the chi-square test $\left(\chi^{2}\right)$. All statistical analyses were performed using the Statistical Package for Social Science SPSS 22.0. Statistical significance was established at the $p<0.05$ level.

\section{Results}

From the total number of children involved in the study, 35 were children with ET, 26 with MEl, and 32 were healthy children as a control group. The study included 93 children, of whom 57 (61.3\%) were boys and 36 (38.7\%) girls. There was no significant difference 

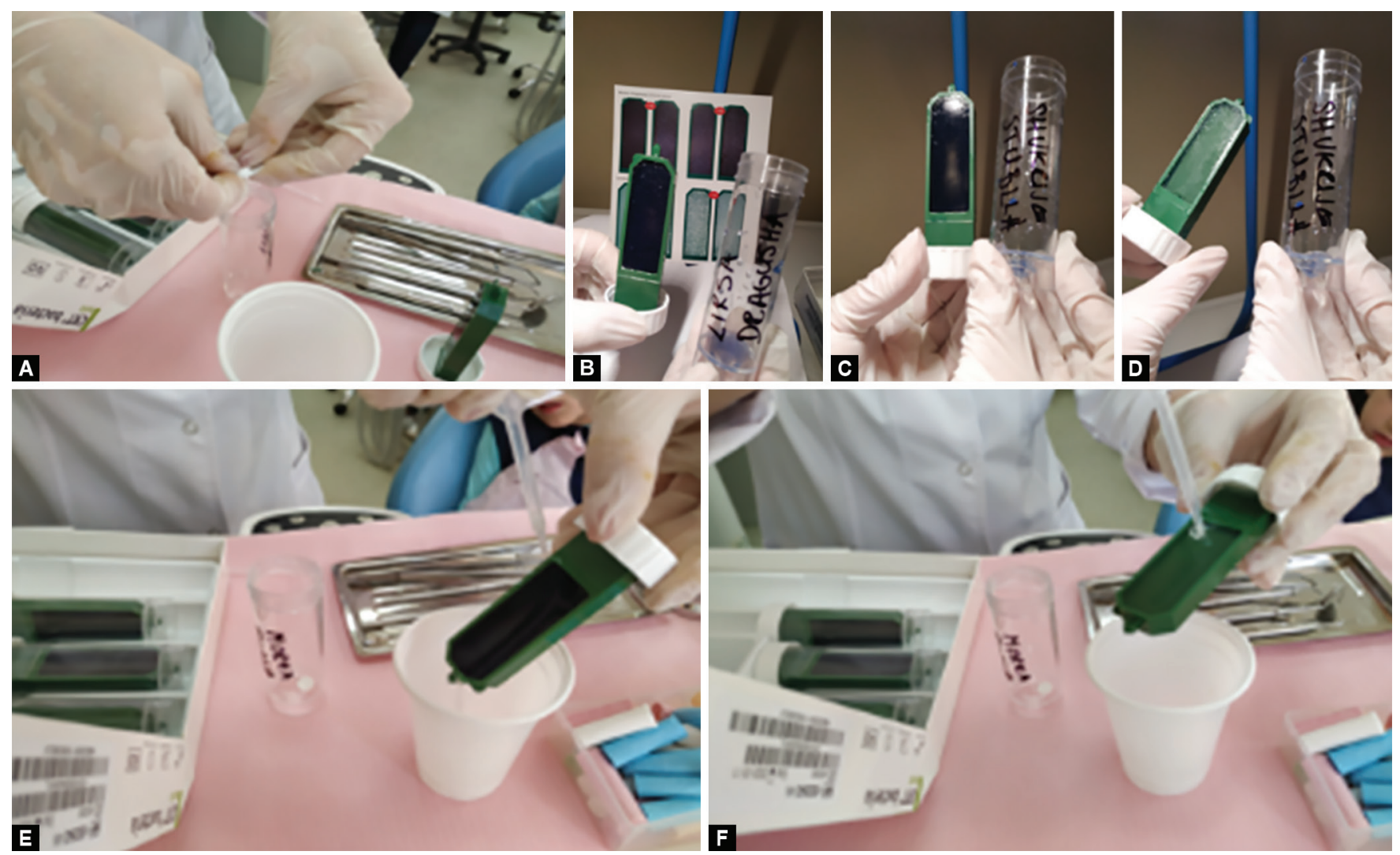

Figs $1 \mathrm{~A}$ to F: (A) Placement of $\mathrm{NaHCO}_{3}$ tablet in the dish of the culture agar; (B) Mutans streptococci CFU/mL saliva reading; (C) Mutans streptococci colony after incubation; (D) Lactobacillus colony after incubation; (E) Dispersion of the saliva sample through the culture media (agar) for mutans streptococci using pipettes; (F) Dispersion of the saliva sample through the culture media (agar) for lactobacilli using pipettes

between groups in terms of gender ratios ( $p=0.504)$, but with a statistically significant difference between age in the three groups $(p<0.001)$ (Table 1). The children included in our research were between ages 5 years to 15 years. The average age of children with ET group was 7.8 years (DS \pm 2.62 years), with MEl was 5.7 years (DS \pm 1.15 years), while those of the control group was 10.6 years (DS \pm 2.05 years) (Table 1 ).

In addition, there were no significant differences between the groups in the variables, such as using fluoride paste, visits to dentist, family income, $(p>0.05)$, with the exception of family education level, method of feeding, use of pacifier, sweet eating habits, toothbrushing, saliva secretion, and rate of oral breathing $(p<0.001)$ (Table 1).

After the evaluation of the percentage of patients with $\mathrm{SM}<$ or $>10^{5} \mathrm{CFU}$ and LB $<$ or $>10^{5} \mathrm{CFU}$ and the comparison between groups are noted that group $\mathrm{Gl}$ has been found to have more streptococci compared to GIl and the control group, whereas GII has been found to have less LB compared to the other two groups (Table 2).

The statistical analysis shows a significant difference in the $\mathrm{OHI}-\mathrm{S}$ index between groups. More cases with the high oral hygiene index score were detected among children with ET (Table 3).

The statistical investigation demonstrated an expressively higher Löe-Silness Gl in the study group, especially in the ET group. The GIl group has been found to have more cases with 0 score of Löe-Silness Gl, both for primary as well as for permanent dentition compared to other groups (Table 4).

\section{Discussion}

This comparative observational study aimed to evaluate the oral health, salivary flow, and specific amount of Streptococcus mutans and LB in saliva between the groups with MEI, and with ET (control groups) and the healthy children group. In terms of outcomes, control groups appear to be more likely to develop a higher OHI-S and Löe-Silness Gl, compared to the healthy group. Moreover, the same groups have a significantly higher risk of developing S. mutans and Lactobacillus, $>10^{5} \mathrm{CFU}$, compared to the healthy group. Also, statistical differences were distinguished for the salivary flow rate, between healthy cases, and control groups.

There are different views in the literature on the role of socioeconomic status and family educational level in oral and microbiological health.

Our study showed a significant difference between subjects in terms of socioeconomic status and family educational level regarding oral and microbiological health.

At the same time, different authors ${ }^{4,7,14,16}$ reported the impact of family education, and socioeconomic status in oral health, and prevalence of dental caries while in the opposite, a study by Bakhshaee et al. ${ }^{18}$ report that there were no discrepancies amongst the study groups, in terms of socioeconomic status, while there was a difference between the DMFTs. This is probably because of drug therapies for allergic rhinitis, which decreases salivary flow rate and may play a role in DMFT. 19,20

In our study, children with ET mostly oral breathers, have had higher plaque index compared with the other two groups, our 
Table 1: Demographic, nutritional, and hygiene habits data in research groups

\begin{tabular}{|c|c|c|c|c|}
\hline & Intervention GI ET, n (\%) & Intervention GII MEI, n (\%) & Control group GIII, n (\%) & $p$ \\
\hline Age (mean \pm SD) & $7.8 \pm 2.62$ & $5.73 \pm 1.15$ & $10.56 \pm 2.05$ & $<0.0001$ \\
\hline \multicolumn{5}{|l|}{ Sex } \\
\hline Male & $23(65.7)$ & $17(65.4)$ & $17(53.1)$ & \multirow[t]{2}{*}{0.504} \\
\hline Female & $12(34.3)$ & $9(34.6)$ & $15(46.9)$ & \\
\hline \multicolumn{5}{|l|}{ Family education level } \\
\hline Primary school & $10(28.6)$ & $10(38.5)$ & $2(6.3)$ & \multirow[t]{3}{*}{0.003} \\
\hline High school & $21(60.0)$ & $13(50.0)$ & $17(53.1)$ & \\
\hline Higher education level & $4(11.4)$ & $3(11.5)$ & $13(40.6)$ & \\
\hline \multicolumn{5}{|l|}{ Family income } \\
\hline$<200$ & $4(11.4)$ & $4(15.4)$ & $0(0.0)$ & \multirow[t]{3}{*}{0.010} \\
\hline $200-450$ & $20(57.1)$ & $19(73.1)$ & $13(40.6)$ & \\
\hline$>450$ & $11(31.4)$ & $3(11.5)$ & 19 (59.4) & \\
\hline \multicolumn{5}{|l|}{ Using fluoride paste } \\
\hline Yes & $9(25.7)$ & $6(23.1)$ & $11(34.4)$ & \multirow[t]{3}{*}{0.683} \\
\hline No & $20(57.1)$ & $14(53.8)$ & $13(40.6)$ & \\
\hline Do not know & $6(17.1)$ & $6(23.1)$ & $8(25.0)$ & \\
\hline \multicolumn{5}{|l|}{ Teeth brush } \\
\hline Once a day & $15(42.9)$ & $16(61.5)$ & $14(43.8)$ & \multirow[t]{3}{*}{0.003} \\
\hline 2-3 per day & $14(40.0)$ & $3(11.5)$ & $17(53.1)$ & \\
\hline Few times in the week & $6(17.1)$ & $7(26.9)$ & $1(3.1)$ & \\
\hline \multicolumn{5}{|l|}{ Visits to dentist } \\
\hline Once in 6 months & $2(5.7)$ & $1(3.8)$ & $1(3.1)$ & \multirow[t]{3}{*}{0.267} \\
\hline Once in the year & $4(11.4)$ & $0(0.0)$ & $10(31.3)$ & \\
\hline Only when has pain & $29(82.9)$ & $25(96.2)$ & $21(65.6)$ & \\
\hline \multicolumn{5}{|l|}{ Sweet eating habits } \\
\hline Often & $18(51.4)$ & $15(57.7)$ & $24(75.0)$ & \multirow[t]{3}{*}{0.008} \\
\hline Rare & $5(14.3)$ & $3(11.5)$ & $7(21.9)$ & \\
\hline Sometimes & $12(34.3)$ & $8(30.8)$ & $1(3.1)$ & \\
\hline \multicolumn{5}{|l|}{ Saliva secretion } \\
\hline Normal & $14(40.0)$ & $15(57.7)$ & $12(37.5)$ & \multirow[t]{3}{*}{$<0.0001$} \\
\hline High & $10(28.6)$ & $0(0.0)$ & $19(59.4)$ & \\
\hline Low & $11(31.4)$ & $11(42.3)$ & $1(3.1)$ & \\
\hline \multicolumn{5}{|l|}{ Method of feeding } \\
\hline Brestfeeding & $10(28.6)$ & $16(61.5)$ & $27(84.4)$ & \multirow[t]{3}{*}{$<0.0001$} \\
\hline Bottle feeding & $22(62.9)$ & $3(11.5)$ & $2(6.3)$ & \\
\hline Both & $3(8.6)$ & $7(26.9)$ & $39.4)$ & \\
\hline \multicolumn{5}{|l|}{ Prolonged pacifier use } \\
\hline Yes & $23(65.7)$ & $4(15.4)$ & $17(53.1)$ & \multirow[t]{2}{*}{$<0.0001$} \\
\hline No & $12(34.3)$ & $22(84.6)$ & $15(46.9)$ & \\
\hline \multicolumn{5}{|l|}{ Mouth breath } \\
\hline Yes & $35(100.0)$ & $14(53.8)$ & $0(0.0)$ & \multirow[t]{2}{*}{$<0.0001$} \\
\hline No & $0(0.0)$ & $12(46.2)$ & $32(100.0)$ & \\
\hline
\end{tabular}

$\mathrm{ET}$, enlarged tonsils; MEl, middle ear infection; SD, standard deviation

results are in concordance with other authors studies too. ${ }^{17,21,22}$ Furthermore, it appears that the child has greater contact with airflow, during respiration and subsequently, gingival inflammation and irritation may be associated with superficial dehydration and decreased salivary flow. Also, a study by Mehta et al. ${ }^{22}$ stated that children who breathe orally appear to be predisposed to form the highest level of dental plaque.
Meanwhile, in our study, the group with tonsillar hypertrophy demonstrated a significant difference compared to healthy group as far as $\mathrm{OHI}-\mathrm{S}$ index values.

In a study by Eryaman et al. ${ }^{5}$ conducted in Turkey amongst 80 children aged $3-12$ years (38 in the intervention group and 42 in the control group), the correlation between poor oral hygiene and tonsillar hypertrophy dmft index was analyzed for 
Table 2: Microbiological status of colonies of Streptococcus mutans and Lactobacillus in research groups

\begin{tabular}{lcrll}
\hline & $\begin{array}{l}\text { Intervention } \\
\text { Intervention } \\
\text { GII MEI, } n \text { (\%) } \\
(\%)\end{array}$ & $\begin{array}{l}\text { Control } \\
\text { group GIII, } \\
n(\%)\end{array}$ & $p$ \\
\hline Lactobacillus & & & & \\
$\mathrm{CFU} / \mathrm{mL}<10^{5}$ & $2(7.4)$ & $20(76.9)$ & $4(12.9)$ & $<0.0001$ \\
$\mathrm{CFU} / \mathrm{mL}>10^{5}$ & $25(92.6)$ & $6(23.1)$ & $27(87.1)$ & \\
Streptococcus mutans & & & \\
$\mathrm{CFU} / \mathrm{mL}<10^{5}$ & $13(37.1)$ & $22(84.6)$ & $17(53.1)$ & 0.001 \\
$\mathrm{CFU} / \mathrm{mL}>10^{5}$ & $22(62.9)$ & $4(15.4)$ & $15(46.9)$ & \\
\hline
\end{tabular}

$\mathrm{ET}$, enlarged tonsils; MEl, middle ear infection; CFU, colony-forming unit

Table 3: OHI-S status of permanent dentition in research groups

\begin{tabular}{|c|c|c|c|c|}
\hline & $\begin{array}{l}\text { Intervention } \\
\text { GI ET, n (\%) }\end{array}$ & $\begin{array}{l}\text { Intervention } \\
\text { GII MEI, } n \\
\text { (\%) }\end{array}$ & $\begin{array}{l}\text { Control } \\
\text { group GIII, } \\
n(\%)\end{array}$ & $p$ \\
\hline \multicolumn{5}{|c|}{ OHI-S permanent dentition } \\
\hline 0 & $7(20.0)$ & $22(84.6)$ & $3(9.4)$ & $<0.0001$ \\
\hline 1 & $9(25.7)$ & $1(3.8)$ & $23(71.9)$ & \\
\hline 2 & $13(37.1)$ & $3(11.5)$ & $5(15.6)$ & \\
\hline 3 & $6(17.1)$ & $0(0.0)$ & $1(3.1)$ & \\
\hline
\end{tabular}

$\mathrm{ET}$, enlarged tonsils; $\mathrm{MEI}$, middle ear infection; $\mathrm{OHI}-\mathrm{S}$, oral health indexsimplified; the scores of $0-1$ were classified as low, and of 2-3 as high oral hygiene index (OHI-S)

Table 4: Löe-Silness gingival index status of primary and permanent dentition in research groups

\begin{tabular}{|c|c|c|c|c|}
\hline & $\begin{array}{l}\text { Intervention } \\
G I E T, n(\%)\end{array}$ & $\begin{array}{l}\text { Intervention } \\
\text { Gll MEI, } n \\
(\%)\end{array}$ & $\begin{array}{l}\text { Control } \\
\text { group GIII, } \\
n(\%)\end{array}$ & $p$ \\
\hline \multicolumn{5}{|c|}{ Primary dentition Löe-Silness GI } \\
\hline 0 & $9(25.7)$ & $19(73.1)$ & $14(43.8)$ & 0.014 \\
\hline 1 & $19(54.3)$ & $6(23.1)$ & $15(46.9)$ & \\
\hline 2 & $6(17.1)$ & $1(3.8)$ & $3(9.4)$ & \\
\hline 3 & $1(2.9)$ & $0(0.0)$ & $0(0.0)$ & \\
\hline \multicolumn{5}{|c|}{ Permanent dentition Löe-Silness GI } \\
\hline 0 & $7(20.0)$ & $22(84.6)$ & $4(12.5)$ & $<0.0001$ \\
\hline 1 & $15(42.9)$ & $1(3.8)$ & $23(71.9)$ & \\
\hline 2 & $11(31.4)$ & $3(11.5)$ & $5(15.6)$ & \\
\hline 3 & $2(5.7)$ & $0(0.0)$ & $0(0.0)$ & \\
\hline
\end{tabular}

ET, enlarged tonsils; $\mathrm{MEI}$, middle ear infection; $\mathrm{Gl}$, gingival index; the scores of $0-1$ were classified as low, and of $2-3$ as high Löe-Silness gingival index (Gl)

the primary dentition, DMFT index for permanent teeth, and the $\mathrm{OHI}-\mathrm{S}$ index. Results of this research have demonstrated a strong correlation between poor oral hygiene and tonsillar hypertrophy. ${ }^{5}$ These findings state that the dental pathogens, S. mutans, and Streptococcus sobrinus, may persuade the immunologic response progression in the tonsils, and accordingly, the question is would the chronic incentive of poor oral hygiene cause hypertrophy of the tonsils. ${ }^{5}$

The current research concludes that in comparison to healthy controls, children with MEl are reported to have higher OHI-S index, Löe-Silness $\mathrm{Gl}$, increased mean CFU of SM and LB, especially in the primary dentition.
Whether there is a correlation between oral health and dental caries in children with MEI has also been investigated by some authors. ${ }^{23-25}$ Kashyap et al. ${ }^{23}$ demonstrated that the risk of early childhood caries was found to be significantly higher in children who were diagnosed with MEl. In their study, the group with middle ear infected children showed a mean CFU of 5.6013, which differed significantly ( $p$ value $=0.001$ ) from noninfected children group. On the contrary, authors Eryaman et al. ${ }^{24}$ and Nelson et al. ${ }^{25}$ conclude from their clinical study that there was no statistically significant association between oral hygiene and the MEI.

They did not find any difference between the group with high $\mathrm{OHI}-\mathrm{S}$ and low OHI-S in connection with present MEls.

The results of our study are consistent with previously published works ${ }^{26-29}$ concerning the relationship between poor oral hygiene and MS and LB colonies. Meanwhile, in a study carried out by Zhou et al., ${ }^{27}$ who found that colonization of MS in 8-32-month-old children was associated with poor oral hygiene, while Köhler et al. ${ }^{28}$ stated that earlier colonization by MS in young children generally translates into increased caries rates. Also, in a study done by Kõll-Klais et al., ${ }^{29}$ a positive correlation was found between the DMFS counts, and the LB counts in Estonian schoolchildren.

\section{Limitations of the Study}

A few limitations should be noted. The main weakness of this paper was the short duration of the study, along with the small number of patients, factors that might have had an effect on the outcome. Another disadvantage is that we only identified the cases we did not follow their treatment outcomes.

\section{Conclusion}

Our results demonstrated that patients with ET and MEI had more initial caries, gingival inflammation, dental plaque, a lower stimulated salivary secretion rate, and an increase in the level of salivary MS and LB than healthy individuals. The future study should be done in a large sampling and should be undertaken in order to obtain relevant data regarding the treatment of children with upper respiratory infections and poor oral health.

\section{Clinical Significance}

Results from our study will help not only the pediatric dentistry specialists but also the other medical professionals like pediatricians, ENT specialists, and nurses to increase their awareness regarding the importance of the overall dental health in children with an ear infection or tonsillitis.

\section{Acknowledgment}

It is a pleasure to thank LTC Elaine Berkowitz, DDS (RET), (US Army, $\mathrm{MC}$ ) for her valuable suggestions and technical assistance.

\section{References}

1. Gomes-Filho IS, Passos JS, da Cruz SS. Respiratory disease and the role of oral bacteria. J Oral Microbiol 2010;2:5811. DOI: 10.3402/jom. v2i0.5811.

2. Paju S, Bernstein JM, Haase EM, et al. Molecular analysis of bacterial flora associated with chronically inflamed maxillary sinuses. J Med Microbiol 2003;52(Pt 7):591-597. DOI: 10.1099/jmm.0.05062-0.

3. Zhou Y, Jiang S, Li KY, et al. Association between oral health and upper respiratory tract infection among children. Int Dent J 2018;68(2): 122-128. DOI: 10.1111/idj.12335. 
4. Duncan B, Ey J, Holberg CJ, et al. Exclusive breast feeding for at least 4 months protects against otitis media. Pediatrics 1993;91(5):867-872.

5. Eryaman E, Oter Ilhan B, Erdinc A. Any relation between poor oral hygiene and tonsillar hypertrophy? KBB-Forum 2013;12(1):1-4.

6. Singh S, Vijayakumar N, Priyadarshini HR, et al. Prevalence of early childhood caries among 3-5 year old pre-schoolers in schools of Marathahalli, Bangalore. Dent Res J (Isfahan) 2012;9:710-714.

7. Edwardsson S, Koch G, Obrink M. Strep. sanguis, Strep. mutans and Strep. salivarius in saliva. Prevalence and relation to caries increment and prophylactic measures. Odontol Revy 1972;23(3):279-296.

8. Köhler B, Andréen I. Mutans streptococci and caries prevalence in children after early maternal caries prevention: a follow-up at 19 years of age. Caries Res 2012;46(5):474-480. DOI: 10.1159/000339665.

9. Marsh PD. Microbiology of dental plaque biofilms and their role in oral health and caries. Dent Clin North Am 2010;54(3):441-454. DOI: 10.1016/j.cden.2010.03.002.

10. Seki M, Karakama F, Terajima T, et al. Evaluation of mutans streptococci in plaque and saliva: correlation with caries development in preschool children. J Dent 2003;31(5):283-290. DOI: 10.1016/S03005712(03)00033-2.

11. Flanagan D. Inspissated oral secretions and a review of their clinical, biological, and physiological significance. Spec Care Dentist 2012;32(3):99-104. DOI: 10.1111/j.1754-4505.2012.00241.x.

12. Ersin NK, Gülen F, Eronat N, et al. Oral and dental manifestations of young asthmatics related to medication, severity and duration of condition. Pediatr Int 2006;48(6):549-554. DOI: 10.1111/j.1442200X.2006.02281.x.

13. WHO: Oral Health Country, Area Profile Programme Oral Hygiene Indices, (on web) http:/www.whocollob.od.mah.se/expl/ohiintrod. html.

14. WHO: World Oral Health County, Area Profile World Health Organization Oral Health Surveys. Basic Methods, 4th ed., Geneva: World Health Organization; 1997.

15. Löe $H$. The gingival index, the plaque index and the retention index systems. J of Periodontology 1967;38(6 Part II):610-616. DOI: 10.1902/ jop.1967.38.6_part2.610.

16. Simplified Oral Hygiene Index|OHI-S—Oral Health Database, Malmö University; 1964.
17. Mummolo S, Nota A, Caruso S, et al. Salivary markers and microbial flora in mouth breathing late adolescents. BioMed Res Int 2018;2018:8687608. DOI: 10.1155/2018/8687608.

18. Bakhshaee $M$, Ashtiani SJ, Hossainzadeh $M$, et al. Allergic rhinitis and dental caries in preschool children. Dent Res J 2017;14(6):376-381. DOI: 10.4103/1735-3327.218560.

19. Wongkamhaeng K, Poachanukoon O, Koontongkaew S. Dental caries, cariogenic microorganisms and salivary properties of allergic rhinitis children. Int J Pediatr Otorhinolaryngol 2014;78(5):860-865. DOI: 10.1016/j.ijporl.2014.03.001.

20. Widmer RP. Oral health of children with respiratory diseases. Paediatr Respir Rev 2010;11(4):226-232. DOI: 10.1016/j.prrv.2010.07.006.

21. Niklander S, Veas L, Barrera C, et al. Risk factors, hyposalivation and impact of xerostomia on oral health-related quality of life. Brazilian Oral Research 2017;31:e14. DOI: 10.1590/1807-3107bor-2017. vol31.0014.

22. Mehta A, Sequeira PS, Sahoo RC, et al. Is bronchial asthma a risk factor for gingival diseases? A control study. NY State Dent J 2009;75(1):44-46.

23. Kashyap N, Katlam T, Avinash A, et al. Middle ear infection in children and its association with dental caries. Medicine and Pharmacy Reports 2019;92(3):271.

24. Eryaman E, Oter Ilhan B, Erdinc A. Poor oral hygiene and middle ear infections: any relationship? Indian J Otolaryngol Head Neck Surg 2013;65(2):173-176. DOI: 10.1007/s12070-012-0616-4.

25. Nelson S, Nechvatal N, Weber J, et al. Dental caries and ear infections in preschool-aged children. Oral Health Prev Dent 2005;3(3):165-171.

26. Mummolo S, Tieri M, Tecco S, et al. Clinical evaluation of salivary indices and levels of streptococcus mutans and Lactobacillus in patients treated with occlus-o-guide. Eur J Paediatr Dent 2014;15(4):367-370.

27. Zhou Y, Yang JY, Zhi QH, et al. Factors associated with colonization of streptococcus mutans in 8-32-month-old children: a cohort study. Aust Dent J 2013;58(4):507-513. DOI: 10.1111/adj.12113.

28. Köhler B, Andréen I, Jonsson B. The earlier the colonization by mutans streptococci, the higher the caries prevalence at 4 years of age. Oral Microbiol Immunol 1988;3(1):14-17. DOI: 10.1111/j.1399-302X.1988. tb00598.x.

29. Kõll-Klais P, Mändar R, Leibur E, et al. High levels of salivary lactobacilli in Estonian school children. Eur J Paediatr Dent 2004;5(2):107-109. 


\section{Questionnaire for Parents and Children Dental Examination}

1. General data

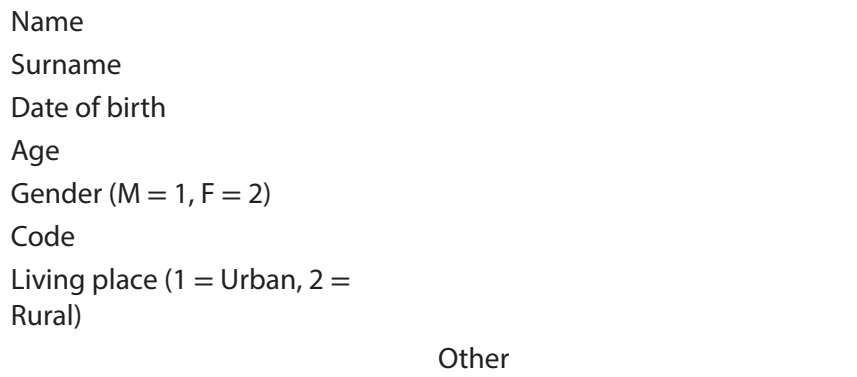

2. Dental/oral examination

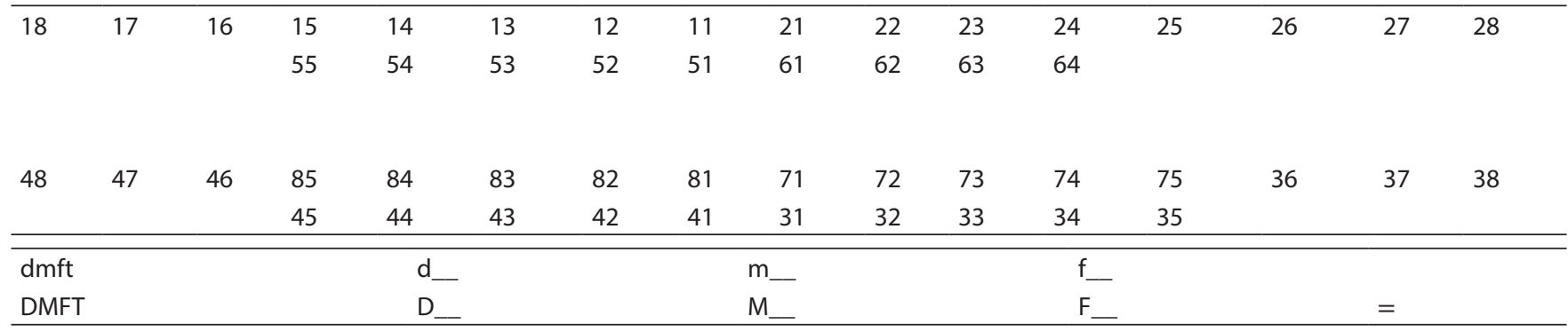
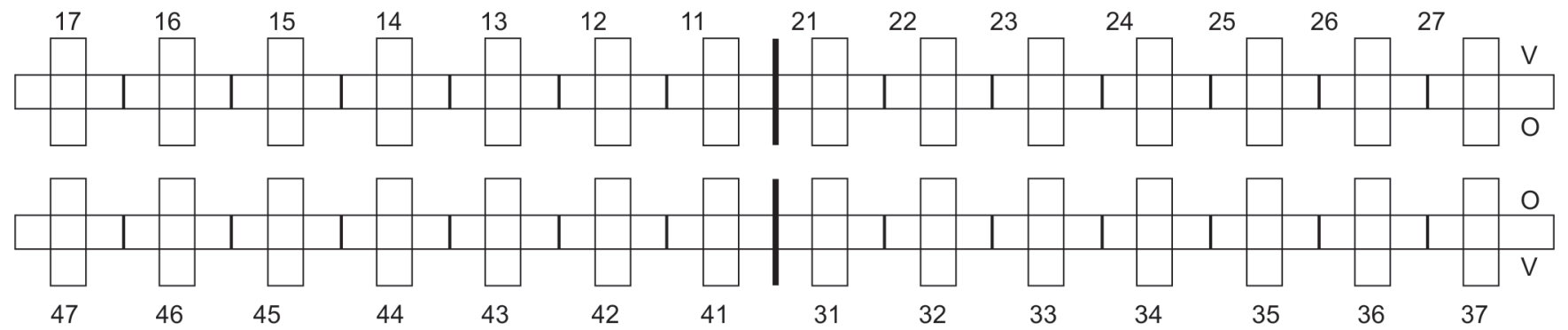

Gingival index-permanent dentition (Löe-Silness)

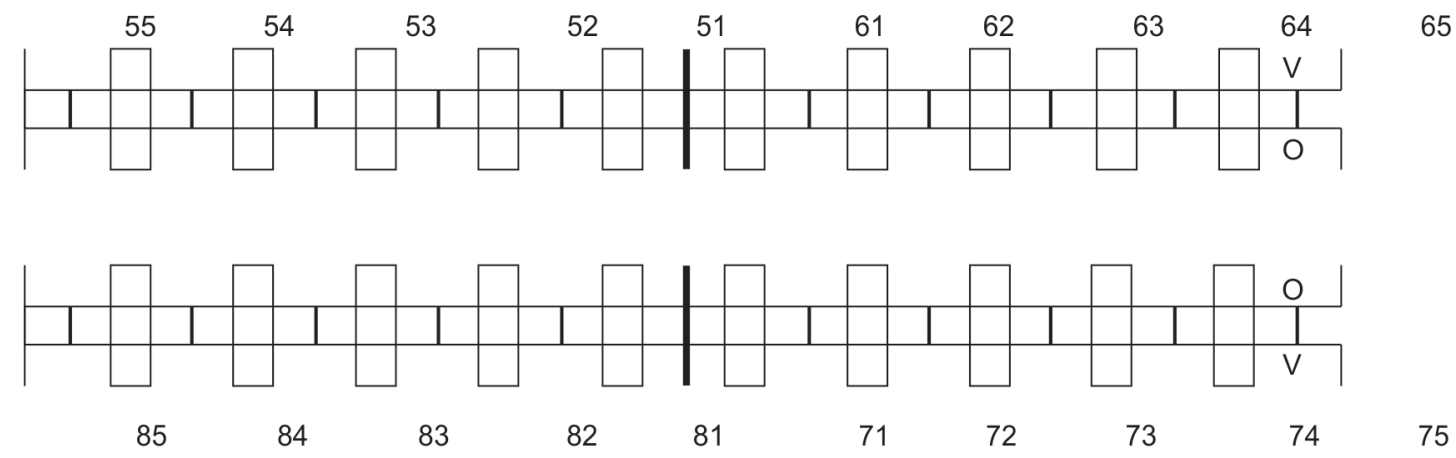

Gingival index-primary dentition (Löe-Silness) 


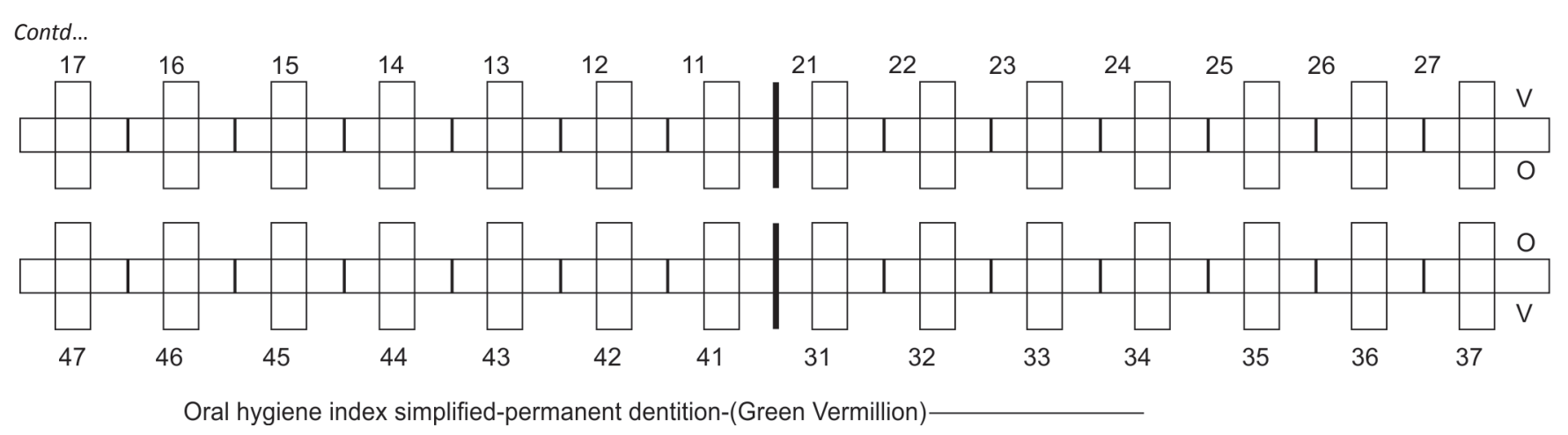

Sagittal direction disarrangement:

\begin{tabular}{l}
\hline I I \\
Transversal direction disarrangement: PO JO \\
Vertical direction disarrangement: \\
\hline Open Deep \\
\hline
\end{tabular}

\begin{tabular}{lll}
\hline Sweet eating habits? & & \\
Often & Yes & No \\
Rare & Yes & No \\
Sometimes & Yes & No \\
Saliva secretion & & \\
Normal & Yes & No \\
High & Yes & No \\
Low & Yes & No \\
Method of feeding? & & \\
Breastfeeding & Yes & No \\
Bottle feeding & Yes & No \\
Both & Yes & No \\
Prolonged pacifier use? & \\
& Yes & \\
& No & \\
Mouth breath? & & \\
& Yes & \\
Note: & No & \\
\hline
\end{tabular}

\begin{tabular}{lll}
\hline \multicolumn{2}{l}{ Family education level? } & \\
Primary school & Yes & No \\
High school & Yes & No \\
Higher education & Yes & \\
level & & \\
Family income? & & No \\
$<200$ Euro & Yes & No \\
200-450 Euro & Yes & No \\
$>450$ Euro & Yes & \\
Using fluoride paste? & & \\
& Yes & \\
& No & \\
Teeth brush & Don't know & \\
Once a day & Yes & No \\
2-3 per day & Yes & No \\
Few times in a week & Yes & No \\
Visit to dentist? & & \\
Once in six months & Yes & No \\
Once in the year & Yes & No \\
Only when has pain & Yes & No \\
\hline
\end{tabular}

Examination date:

Place: 